\title{
SINGULAR INTEGRALS AND ESTIMATES FOR THE CAUCHY-RIEMANN EQUATIONS
}

\author{
BY E. M. STEIN \\ Communicated August 15, 1972
}

1. Introduction. Our purpose here is to give new estimates for the inhomogeneous Cauchy-Riemann equations $\bar{\partial} u=f$, in a smooth strictly pseudo-convex domain in $C^{n}$. The estimates we shall present should not, however, be regarded as isolated calculations; they are part of a larger pattern of results which arise from adopting the following general point of view ${ }^{1}$ : There is an intimate connection between some new singular integrals that arise (and the estimates to be made for them) in the following areas:

(1) In the context of nilpotent groups, motivated partly by the study of intertwining operators. See [5], [8], and [1] and $\S 7$ below.

(2) The singular integrals that occur in the solution of the $\bar{\partial} u=f$ problem, as given by Grauert and Lieb [2], Henkin [3], and Kerzman [4].

(3) Boundary behavior of holomorphic functions of several complex variables. See $[12]$ and $\S 5$ below.

(4) Singular integrals related to the Bergman kernel. See $\S 6$.

From the qualitative point of view what is common to these areas (and distinguishes them from the more classical integrals and estimates) is the splitting of directions at each point, together with the nonisotropic way that singularities behave and estimates are made. The nonisotropy is not always apparent for $L^{p}$ estimates, but its role becomes clear when calculating with the appropriate Lipschitz (or Hölder) inequalities. It is with the latter type of estimates that we are principally concerned here. Detailed proofs and further results will be given at another occasion.

2. Standard Lipschitz spaces. Recall the definition of $\Lambda_{\alpha}(R), 0<\alpha<\infty$, as given in [11, Chapter V]. From it we can define the corresponding spaces $\Lambda_{\alpha}(I)$, for the unit interval $I=[0,1]$ in the usual way, as the Banach space obtained by restrictions, i.e., as the quotient $\Lambda_{\alpha}(R) / \mathscr{I}$, where $\mathscr{I}=\left\{f \in \Lambda_{\alpha}(\boldsymbol{R}): f(t)=0, t \in I\right\}$.

Next, suppose $\mathscr{R}$ is a smooth Riemannian manifold which for simplicity we assume is isometrically embedded in Euclidean $\boldsymbol{R}^{N}$. For each integer $l$ we define the class of curves $\mathscr{C}^{l}$ in $\mathscr{R}$ to be $\mathscr{C}^{l}=\{x(t): I \ni t \rightarrow x(t) \in \mathscr{R}$, $\left.\left|x^{\prime}(t)\right| \leqq 1, \ldots,\left|x^{(l)}(t)\right| \leqq 1\right\}$. We can now define $\Lambda_{\alpha}(\mathscr{R})$. Let $l$ be smallest

AMS(MOS) subject classifications (1970). Primary 44A25, 35N15, 26A16; Secondary $43 \mathrm{~A} 80$.

${ }^{1}$ Further background for this approach may be found in $[10]$. 
integer $>\alpha$. Then a complex-valued function $u$ on $\mathscr{R}$ is in $\Lambda_{\alpha}(\mathscr{R})$ if for each curve $x(\cdot)$ in $\mathscr{C}^{l}$, the function $t \rightarrow u(x(t))$ belongs to $\Lambda_{\alpha}(I)$. We take as norm

$$
\|u\|_{\Lambda_{\alpha}(\mathscr{R})}=\sup _{x(\cdot) \in \mathscr{C} l}\|u(x(\cdot))\|_{\Lambda_{\alpha}(I)} .
$$

It can be shown that the definition given here, in the special case $\mathscr{R}=\boldsymbol{R}^{n}$, is consistent with that given in [11].

3. Some new Lipschitz spaces. We now modify the above definitions so as to take into account the nonisotropy which is crucial in what follows. For each $x \in \mathscr{R}$ let $T_{x}$ denote the tangent space at $x$. Assume that there is given a direct sum decomposition $T_{x}=T_{x}^{1} \oplus T_{x}^{2}$, with the property that the mappings $x \rightarrow T_{x}^{i}$ are smooth. We give two basic examples of this. $\mathscr{D}$ is an open subdomain of $\boldsymbol{C}^{n}$ with smooth boundary.

EXAMPLE $1 . \mathscr{R}=\partial \mathscr{D}$. For each $x \in \partial \mathscr{D}$, let $v_{x}$ be the unit normal vector at $x$. Set $T_{x}^{2}=\left\{\boldsymbol{R} i v_{x}\right\}$, and $T_{x}^{1}$ the orthogonal complement of $T_{x}^{2}$ in $T_{x}$.

EXAMPLE 2. $\mathscr{R}=\mathscr{D}$. Define a smooth mapping $x \rightarrow \bar{x}$ of $\mathscr{D}$ to $\partial \mathscr{D}$ with the property that whenever $x$ is close to $\partial \mathscr{D}$ then $\bar{x}$ is the normal projection of $x$ to $\partial \mathscr{D}$. Set $T_{x}^{2}=\left\{\boldsymbol{C} v_{\bar{x}}\right\}$, and $T_{x}^{1}$ the orthogonal complement of $T_{x}^{2}$ in $T_{x}$. For these examples see also [12, pp. 34 and 55].

Return to the general case. For $0<\alpha<\infty$ we define the space $\Gamma_{\alpha}(\mathscr{R})$ to consist of those functions $u$ which are in $\Lambda_{\alpha}(\mathscr{R})$, but satisfy the additional property that when restricted to curves whose tangents lie in $T_{x}^{1}$, these functions are in $\Lambda_{2 \alpha}$. More precisely, let $l$ be the smallest integer $>2 \alpha$. Then for norm we take

$$
\|u\|_{\Gamma_{\alpha}(\mathscr{R})}=\|u\|_{\Lambda_{\alpha}(\mathscr{R})}+\sup \left\{\|u(x(\cdot))\|_{\Lambda_{2 \alpha}(I)}\right\},
$$

where the "sup" is taken over all curves $x(\cdot)$ which belong to $\mathscr{C}^{l}$ and have the property that $x^{\prime}(t) \in T_{x(t)}^{1}, t \in I$.

These definitions can be considerably generalized but we shall not pursue this point here. We make some remarks about the spaces $\Lambda_{1}(\mathscr{R})$ and $\Gamma_{1 / 2}(\mathscr{R})$. It is well known that whenever $f \in \Lambda_{1}(I),|f(t+h)-f(t)|$ $\leqq A|h| \log (1 /|h|)$, as $h \rightarrow 0$, but no better estimate involving the first difference can be made for this class. ${ }^{2}$ With this in mind we define $\hat{\Lambda}_{1}(\mathscr{R})$ to be the class of bounded $u$ so that $|u(x)-u(y)| \leqq A|x-y| \log (1 /|x-y|)$, whenever $|x-y| \leqq \frac{1}{2}$; we give $\tilde{\Lambda}_{1}(\mathscr{R})$ an obvious norm. Clearly then $\Lambda_{1}(\mathscr{R}) \subset \tilde{\Lambda}_{1}(\mathscr{R})$. There is a similar situation for $\Gamma_{1 / 2}(\mathscr{R})$. We define $\tilde{\Gamma}_{1 / 2}(\mathscr{R})$ to be the class of all bounded functions $u$ so that

$$
|u(x)-u(y)| \leqq A|x-y|^{1 / 2},
$$

all $x, y \in \mathscr{R}$, and

$$
\left|u\left(x\left(t_{1}\right)\right)-u\left(x\left(t_{2}\right)\right)\right| \leqq A\left|t_{1}-t_{2}\right| \log \left(1 /\left|t_{1}-t_{2}\right|\right)
$$

\footnotetext{
${ }^{2}$ See Zygmund [13, p. 44]. Note, however, that there the $\Lambda_{1}$ spaces are designated as $\Lambda_{*}$.
} 
whenever $\left|t_{1}-t_{2}\right| \leqq \frac{1}{2}$ and $t \rightarrow x(t)$ is a curve in the set $\mathscr{C}^{2}$ whose tangents, $x^{\prime}(t)$, lie in $T_{x(t)}^{1}$. Again $\Gamma_{1 / 2}(\mathscr{R}) \subset \widetilde{\Gamma}_{1 / 2}(\mathscr{R})$.

4. Estimates for $\bar{\partial} u=f$. Let now $\mathscr{D}$ be a smooth bounded domain in $C^{n}$ which is strictly pseudo-convex. Let $f$ be a once continuously differentiable $(0,1)$ form defined in $\overline{\mathscr{D}}$, which satisfies the integrability condition $\bar{\partial} f=0$. The problem is to estimate the smoothness of solutions of $\bar{\partial} u=f$. We consider the splitting described in Example 2 of $\$ 3$, and the resulting $\Gamma(\mathscr{D})$ spaces.

Recent integral representations for $u$ and estimates are due to Grauert and Lieb [2], Henkin [3], and Kerzman [4]. Basing ourselves on these integral representations, then for the resulting linear mapping $f \rightarrow u$ we can prove

THEOREM 1. Suppose $f$ is bounded on $\mathscr{D}$ and $\bar{\partial} f=0$. Then the solution $u \in \widetilde{\Gamma}_{1 / 2}(\mathscr{D}) ;$ also $\|u\|_{\tilde{\Gamma}_{1 / 2}(\mathscr{D})} \leqq A\|f\|_{\infty}$.

An immediate corollary is

COROLlaRY $^{3} . u \in \Lambda_{1 / 2}(\mathscr{D})$, and $\|u\|_{\Lambda_{1 / 2}(\mathscr{D})} \leqq A\|f\|_{\infty}$.

We can write $u=u_{1}+u_{2}$, where $u_{1}$ is an integral over $\mathscr{D}$ of the BochnerMartinelli type. For it we have the classical estimate $u_{1} \in \Lambda_{1}(\mathscr{D})$, and trivially $\Lambda_{1} \subset \Gamma_{1 / 2} \subset \tilde{\Gamma}_{1 / 2} . u_{2}$ is for us the interesting term, and is expressed as an integral over the boundary $\partial \mathscr{D}^{4}$; it involves essentially the "new" singular integrals, and requires for its analysis the appropriate splitting of directions. For $u_{2}$ we prove

LEMMA. Consider the second-order differential monomial $\partial^{2} / \partial \xi_{1} \partial \xi_{2}$ in the directions of the unit vectors $\xi_{1}$ and $\xi_{2}$. Then

$$
\partial^{2} u_{2}(x) / \partial \xi_{1} \partial \xi_{2}=O\left(\delta^{-3 / 2}\right), \quad O\left(\delta^{-1}\right) .
$$

The first alternative holds when $\xi_{1}$ and $\xi_{2}$ are in general position; the second, when at least one of $\xi_{1}$ or $\xi_{2}$ belongs to $T_{x}^{1}$. $\delta$ denotes the distance of $x$ from $\partial \mathscr{D}$.

5. Holomorphic functions. We shall examine further the kind of conditions occurring in (1) above, and show that the $\Gamma_{\alpha}$ spaces are entirely natural in the context of holomorphic functions. Let $\mathscr{D}$ be a bounded smooth domain in $\boldsymbol{C}^{n}$, but here we shall not make any assumption concerning its pseudo-convexity. For any function $u$ in $\mathscr{D}$, consider the conditions

\footnotetext{
${ }^{3}$ For the corollary, see Romanov and Henkin [9].

4 See e.g. the expression for it in [3, p. 277].
} 
(2) $\left|\left(\frac{\partial}{\partial \xi_{1}} \cdots \frac{\partial}{\partial \xi_{k+l}}\right) u(x)\right| \leqq A_{k, l} \delta^{\alpha-k-l / 2}, \quad \alpha>0, \alpha-k-l / 2<0$,

where $k$ of the unit directions are unrestricted, but the remaining $l$ are required to lie in $T_{x}^{1}$. (Actually conditions of this kind need to be imposed for only finitely many $k$ and $l$.)

THEOREM 2. Suppose $u$ is holomorphic in $\mathscr{D}$ and continuous in $\overline{\mathscr{D}}$. Then

$$
u \in \Gamma_{\alpha}(\mathscr{D}) \Leftrightarrow u \text { satisfies (2). }
$$

COROLlaRY 1. $\left.u \in \Gamma_{\alpha}(\mathscr{D}) \Leftrightarrow u\right|_{\partial \mathscr{D}} \in \Gamma_{\alpha}(\partial \mathscr{D})$.

We know by classical estimates that $\left.u \in \Lambda_{\alpha}(\mathscr{D}) \Leftrightarrow u\right|_{\partial \mathscr{D}} \in \Lambda_{\alpha}(\partial \mathscr{D})$. The connection between $\Lambda_{\alpha}$ and $\Gamma_{\alpha}$ is highlighted by

COROllary $2 .{ }^{5} u \in \Gamma_{\alpha}(\mathscr{D}) \Leftrightarrow u \in \Lambda_{\alpha}(\mathscr{D})$.

Thus a holomorphic function $u$ which satisfies the classical $\Lambda_{\alpha}$ Lipschitz condition must also satisfy the stronger $\Gamma_{\alpha}$ condition.

6. Bergman kernel operators for the unit ball. Here $\mathscr{R}=\mathscr{D}=$ unit ball in $\boldsymbol{C}^{n}$, and we consider the projection operator $P$ given by the Bergman kernel of $\mathscr{D}$, namely,

$$
(P u)(z)=\int_{|\xi| \leqq 1} K(z, \zeta) u(\zeta) d \sigma(\zeta),
$$

where $K(z, \zeta)=\left(n ! / \pi^{n}\right)(1-z \cdot \bar{\zeta})^{-n-1}$.

THEOREM 3. The mapping $u \rightarrow P(u)$

(1) takes $\Gamma_{\alpha}(\mathscr{D})$ continuously to $\Gamma_{\alpha}(\mathscr{D}), 0<\alpha<\infty$;

(2) takes $L^{p}(\mathscr{D})$ continuously to $L^{p}(\mathscr{D}), 1<p<\infty$.

The proof of part (1) uses the characterization given in Theorem 2 .

An application is as follows. Let $u$ be any solution of $\bar{\partial} u=f$ in the unit ball. Then Kohn's solution (in [6]) is given by $u_{0}=u-P(u)$, since it is characterized by being orthogonal to holomorphic functions. The theorem then shows that any $\Gamma_{\alpha}$ or $L^{p}$ estimate made for $u$ (such as in $\$ 4$ above) holds also for Kohn's solution, in the case of the unit ball.

7. Nilpotent groups. As we have already indicated, there are several closely related models for the kind of singular integrals we are dealing with. Besides those already discussed there are the "fractional integrals" which we now describe.

\footnotetext{
${ }^{5} \mathrm{I}$ owe the formulation of this corollary to a conversation with P. Malliavin.
} 
Let $X$ be a simply-connected nilpotent Lie group, and let $x \rightarrow|x|$ be a "norm function" on it in the sense of [5]. Thus $|x|>0$ iff $x \neq$ identity, $|x|$ is $C^{\infty}$ in the complement of the identity, $|x|=\left|x^{-1}\right|$, and $d x /|x|$ is invariant under an appropriate one-parameter group of automorphisms of $X$ (dilations). For $\alpha$ positive and sufficiently small it is natural to define the space ${ }^{*} \Gamma_{\alpha}(X)$ to consist of those functions $f$ on $X$ for which

$$
\sup _{x \in X}|f(x y)-f(x)| \leqq A|y|^{\alpha} .
$$

Since $|y|=\left|y^{-1}\right|$, an equivalent definition is obtained if left translation is used in place of right translations.

Let $\Omega$ be a function defined on $X$-\{identity $\}$ which is $C^{\infty}$ there, and homogeneous of degree 0 , i.e., is invariant under the dilations. We shall consider the fractional integrals $I_{\alpha}$ given by

$$
I_{\alpha}(f)(x)=\int_{X} \frac{\Omega(y)}{|y|^{1-\alpha}} f\left(y^{-1} x\right) d y, \quad 0<\alpha<1 .
$$

This can be rewritten as $I_{\alpha}(f)=\int K\left(x y^{-1}\right) f(y) d y$, with $K(x)$ $=\Omega(x) /|x|^{1-\alpha}$.

In order to be able to deal with bounded $f$ (for which (4) may not converge), we modify $I_{\alpha}$ by adding a constant, and define

$$
\tilde{I}_{\alpha}(f)(x)=\int_{X}\left[K\left(x y^{-1}\right)-K\left(y^{-1}\right)\right] f(y) d y .
$$

We let $d$ be the largest positive exponent satisfying the condition that $\|x\| \leqq A|x|^{d}$, when $\|x\| \leqq 1$, where $\|\cdot\|$ is a standard Euclidean norm on $X$. (For the determination of $d$, see [5, p. 498].)

THEOREM 4. (1) If $f$ is bounded, then $\tilde{I}_{\alpha}(f) \in{ }^{*} \Gamma_{\alpha}(X)$, whenever $0<\alpha<d$.

(2) If $f$ is bounded and $f \in{ }^{*} \Gamma_{\beta}(X)$, then $\tilde{I}_{\alpha}(f) \in{ }^{*} \Gamma_{\alpha+\beta}(X)$, whenever $0<\alpha+\beta<d$.

(3) If $f \in L^{p}(X)$, then $I_{\alpha}(f) \in L^{q}(X)$, whenever $1 / q=1 / p-\alpha$, and $1<p<q<\infty$.

Remarks. 1 . When $X=\boldsymbol{R}^{n}$, we can take $|x|=\|x\|^{n}$. Then $d=1 / n$, and ${ }^{*} \Gamma_{\alpha}(X)$ is essentially $\Lambda_{n \alpha}\left(\boldsymbol{R}^{n}\right)$.

2. Closer to the results above we take $X=\{(z, w)\}=C^{n-1} \times \boldsymbol{R}^{1}$, with $\left(z_{1}, w_{1}\right) \cdot\left(z_{2}, w_{2}\right)=\left(z_{1}+z_{2}, w_{1}+w_{2}-2 \operatorname{Im} z_{1} \bar{z}_{2}\right)$. Here we can take $|(z, w)|$ $=\left(|z|^{4}+w^{2}\right)^{n / 2}$, and hence $d=1 / 2 n$. (See also [10, p. 179].)

For $x \in X$ we can define the splitting $T_{x}=T_{x}^{1} \oplus T_{x}^{2}$ as follows. We identify $T_{1}$ with the space $C^{n-1} \times R^{1}=X$ via the exponential map. Take $T_{1}^{1}$ to correspond to $\{(z, 0)\}$, and $T_{1}^{2}$ to correspond to $\{(0, w)\}$. Finally let $T_{x}^{2}$ denote the images of $T_{1}^{2}$ under left translation by $x$. With this splitting, 
the spaces $\Gamma_{n \alpha}(X)$, as defined in $\S 3$, correspond essentially to ${ }^{*} \Gamma_{\alpha}(X)$, whenever $0<\alpha<1 / 2 n$. The extension of the above results to the case $\alpha \geqq d$ is then indicated by the case already treated in $\$ 3$.

3. For the results corresponding to Theorem 4 in the limiting case $\alpha=0$ (which are, however, of a different character), see e.g. Koranyi and Vagi [8].

4. Of further interest is the fact, recently shown by G. Folland [1a], that a fundamental solution of the $\operatorname{Re}\left(\square_{b}\right)$ Laplacian of Kohn [7], corresponding to the boundary of the unit ball, can be realized explicitly as an integral of the form (4).

\section{REFERENCES}

1. R. Coifman and G. Weiss, Analyse harmonique non-commutative sur certains espaces homogenes, Lecture Notes in Math., vol. 242, Springer-Verlag, Berlin and New York, 1971.

1a. G. B. Folland, A fundamental solution for a subelliptic operator, Bull. Amer. Math. Soc. (to appear).

2. H. Grauert and I. Lieb, Das Ramirezsche Integral und die Lösung der Gleichung $\partial f=$ $\alpha$ im Bereich der beschränkten Formen, Complex Analysis, 1969 (Proc. Conf. Rice Univ., Houston, Tex.), Rice Univ. Studies 56 (1970), no. 2, 29-50. MR 42 \# 7938.

3. G. M. Henkin, Integral representation of functions in strictly pseudoconvex domains and applications to the J-problem, Mat. Sb. 82 (124) (1970), 300-308 = Math. USSR Sb. 11 (1970), 273-281. MR 42 \# 534.

4. N. Kerzman, Hölder and $L^{p}$ estimates for solutions of $\bar{\partial} u=f$ in strongly pseudoconvex domains, Comm. Pure Appl. Math. 24 (1971), 301-379. MR 43 \# 7658.

5. A. W. Knapp and E. M. Stein, Intertwining operators for semi-simple groups, Ann. of Math. (2) 93 (1971), 489-578.

6. J. J. Kohn, Harmonic integrals on strongly pseudoconvex manifolds. I, II, Ann. of Math. (2) 78 (1963), 112-148; ibid. (2) 79 (1964), 450-472. MR 27 \#2999; MR 34 \#8010.

7. Boundaries of complex manifolds, Proc. Conf. Complex Analysis (Minneapolis, 1964), Springer, Berlin, 1965, pp. 81-94. MR 30 \# 5334.

8. A. Koranyi and I. Vagi, Singular integrals on homogeneous spaces and some problems of classical analysis, Ann. Scuola Norm. Sup. Pisa 25 (1971), 575-648.

9. A. V. Romanov and G. M. Henkin, Exact Hölder estimates for the solutions of the z-equation, Izv. Akad. Nauk SSSR Ser. Mat. 35 (1971), 1171-1183= Math. USSR Izv. 5 (1971), 1180-1191.

10. E. M. Stein, Some problems in harmonic analysis suggested by symmetric spaces and semi-simple groups, Proc. Internat. Congress Math. (Nice, 1970), vol. 1, Gauthier-Villars, Paris, 1971, pp. 173-189.

11. - Singular integrals and differentiability properties of functions, Princeton, 1970.

12. - Boundary behavior of holomorphic functions of several complex variables, Mathematical Notes, Princeton, 1972.

13. A. Zygmund, Trigonometrical series. Vols. I, II, 2nd ed., Cambridge Univ. Press, New York, 1959. MR 21 \#6498.

Department of Mathematics, Princeton University, Princeton, New Jersey 08540 